\title{
A NOTE ON THE SECOND ORDER UNIVERSALITY AT THE EDGE OF COULOMB GASES ON THE PLANE
}

\author{
DJALIL CHAFAÏ AND SANDRINE PÉCHÉ
}

\begin{abstract}
We consider in this note a class of two-dimensional determinantal Coulomb gases confined by a radial external field. As the number of particles tends to infinity, their empirical distribution tends to a probability measure supported in a centered ring of the complex plane. A quadratic confinement corresponds to the complex Ginibre Ensemble. In this case, it is also already known that the asymptotic fluctuation of the radial edge follows a Gumbel law. We establish in this note the universality of this edge behavior, beyond the quadratic case. The approach, inspired by earlier works of Kostlan and Rider, boils down to identities in law and to an instance of the Laplace method.
\end{abstract}

\section{INTRODUCTION}

Let us consider a gas of charged particles $\left\{z_{1}, \ldots, z_{n}\right\}$ on the complex plane $\mathbb{C}$, confined individually by the external field $Q$ and experiencing a Coulomb pair repulsive interaction. This corresponds to the probability distribution on $\mathbb{C}^{n}$ with density proportional to

$$
\left(z_{1}, \ldots, z_{n}\right) \in \mathbb{C}^{n} \mapsto \prod_{j=1}^{n} e^{-n Q\left(z_{j}\right)} \prod_{1 \leq j<k \leq n}\left|z_{j}-z_{k}\right|^{\beta},
$$

where $\beta>0$ is a fixed parameter and where $Q: \mathbb{C} \rightarrow \mathbb{R}$ is a fixed smooth function. We are mostly interested in asymptotics in $n \rightarrow \infty$ of this particles system. To ensure the integrability for large enough $n$, and following [11, it is convenient to assume that there exists some real number $\beta^{\prime}>\beta$ with $\beta^{\prime} \geq 1$ such that $c:=\sup _{z \in \mathbb{C}}\left\{(1+|z|)^{\beta^{\prime}} e^{-Q(z)}\right\}<\infty$. Indeed, using the inequality $|a-b| \leq(1+|a|)(1+|b|)$ valid for any $a, b \in \mathbb{C}$, we get

$$
\prod_{j=1}^{n} e^{-n Q\left(z_{j}\right)} \prod_{j<k}\left|z_{j}-z_{k}\right|^{\beta} \leq \prod_{j=1}^{n}\left(\left(1+\left|z_{j}\right|\right)^{\beta} e^{-Q\left(z_{j}\right)}\right)^{n} \leq c^{n^{2}} \prod_{j=1}^{n}\left(1+\left|z_{j}\right|\right)^{-n\left(\beta^{\prime}-\beta\right)} .
$$

The factor $n$ in front of $Q$ in the density (1.1) is motivated by the remarkable formula

$$
\sum_{j=1}^{n} n Q\left(z_{j}\right)-\beta \sum_{j<k} \log \left|z_{j}-z_{k}\right|=n^{2}\left(\int Q(z) d \mu_{n}(z)+\frac{\beta}{2} \iint_{z \neq w} \log \frac{1}{|z-w|} d \mu_{n}(z) d \mu_{n}(w)\right) .
$$

where $\mu_{n}:=\frac{1}{n} \sum_{k=1}^{n} \delta_{z_{k}}$ is the empirical distribution of the particles. Indeed, following [2, 17, 1, 11] (see [5] for more general models), on the space $\mathcal{M}_{1}(\mathbb{C})$ of probability measures on $\mathbb{C}$ equipped with the topology of narrow convergence (i.e. the dual topology related to bounded continuous test functions), the functional

$$
\mu \in \mathcal{M}_{1}(\mathbb{C}) \mapsto I_{Q}(\mu)=\frac{1}{2} \iint_{\mathbb{C}^{2}}\left(Q(z)+Q(w)+\beta \log \frac{1}{|z-w|}\right) d \mu(z) d \mu(w)
$$

is strictly convex, bounded from below with compact level sets, admits a unique minimizer $\mu_{Q}$, and the empirical distribution $\mu_{n}=\frac{1}{n} \sum_{k=1}^{n} \delta_{z_{k}}$ satisfies a large deviations principle for the weak topology at speed $n^{2}$ with good rate function $I_{Q}-I_{Q}\left(\mu_{Q}\right)$. In particular, from the

Date: October 2013. Revised April 2014. Accepted in Journal of Statistical Physics.

2000 Mathematics Subject Classification. 82B21.

Key words and phrases. System of particles; Coulomb gases; Extreme values; Gumbel law. 
first Borel-Cantelli lemma, with probability one, $\mu_{n} \rightarrow \mu_{Q}$ weakly as $n \rightarrow \infty$. Following [21, Th. 1.3], it can be shown that $\mu_{Q}$ has compact support when $\lim _{|z| \rightarrow \infty}|z|^{\beta} e^{-Q(z)}=0$.

Remark 1.1 (Random matrices). The Coulomb gas (1.1) is the spectrum of an Ensemble of random normal matrices obtained by conjugating with an independent Haar unitary matrix, see for instance [6, 7, 1. On the other hand, such a Coulomb gas also describes the spectrum of some few non normal random matrix ensembles. For instance, if $A$ is a random $n \times n$ matrix with i.i.d. complex Gaussian entries $\mathcal{N}\left(0, \frac{1}{2 n} I_{2}\right)$ of mean 0 and variance $1 / n$ then its eigenvalues are a Coulomb gas (1.1) with $\beta=2$ and $Q(z)=|z|^{2}$, and in this case $\mu_{Q}$ is the uniform distribution on the unit disc of $\mathbb{C}$, see [8, 13, 4]. Furthermore, if $B$ is an independent copy of $A$, then the spectrum of $A B^{-1}$ is a Coulomb gas (1.1) with $\beta=2$ and $Q(z)=\log \left(1+|z|^{2}\right)$, and in this case, $\mu_{Q}$ is not compactly supported and is actually heavy tailed, see [15, 11]. In both examples, $\beta=2$ and $Q$ is radially symmetric.

We suppose from now on that $Q$ is radially symmetric in the sense that

$$
Q(z)=V(|z|)
$$

where $V: \mathbb{R}_{+} \rightarrow \mathbb{R}$ is smooth and such that either $r \mapsto r V^{\prime}(r)$ is increasing or $V$ is convex, with $r^{\beta} e^{-V(r)} \rightarrow 0$ as $r \rightarrow \infty$. Let $r_{0}$ be the smallest number such that $V^{\prime}(r)>0$ for all $r>r_{0}$, and let $R_{0}$ be the smallest solution of $R_{0} V^{\prime}\left(R_{0}\right)=\beta$. Then $0 \leq r_{0}<R_{0}<\infty$ and, following [21, Th. 6.1], the probability measure $\mu_{Q}$ is, in polar coordinates $\left(z=r e^{i \theta}\right)$,

$$
d \mu_{Q}(z)=\frac{1}{2 \pi \beta}\left(r V^{\prime}(r)\right)^{\prime} \mathbf{1}_{\left[r_{0}, R_{0}\right]}(r) \mathbf{1}_{[0,2 \pi]}(\theta) d r d \theta .
$$

This formula comes in fact from $d x d y=r d r d \theta$ and $r \Delta Q(z)=r V^{\prime \prime}(r)+V^{\prime}(r)=\left(r V^{\prime}(r)\right)^{\prime}$. Let $\left\{z_{1}, \ldots, z_{n}\right\}$ be the Coulomb gas (1.1) (in other words the atoms of $\mu_{n}$ ) and

$$
|z|_{(1)} \geq \cdots \geq|z|_{(n)}
$$

be their reordered moduli (in other words the order statistics of the moduli). In particular,

$$
|z|_{(1)}=\max _{1 \leq k \leq n}\left|z_{k}\right| \text { and }|z|_{(n)}=\min _{1 \leq k \leq n}\left|z_{k}\right| .
$$

Following Rider [18] (see also [20]), in the case $\beta=2$ and $V(r)=r^{2}$ then $|z|_{(1)} \stackrel{\mathbb{P}}{\rightarrow} 1$ as $n \rightarrow \infty$ and the asymptotic fluctuation follows a Gumbel law. The aim of this note is to show, still for $\beta=2$, the universality of this result beyond the quadratic case on $V$. The following structural result forms the basement of all the remaining results of this note.

Theorem 1.2 (Layered structure). If $\beta=2$ then we have the identity in distribution

$$
\left(|z|_{(1)}, \ldots,|z|_{(n)}\right) \stackrel{d}{=}\left(R_{(1)}, \ldots, R_{(n)}\right)
$$

where $|z|_{(1)} \geq \cdots \geq|z|_{(n)}$ are as in (1.2) and where $R_{(1)} \geq \cdots \geq R_{(n)}$ is the order statistics of independent random variables $R_{1}, \ldots, R_{n}$ with $R_{k}$ of density proportional to

$$
t \mapsto t^{2 k-1} e^{-n V(t)} \mathbf{1}_{[0, \infty)}(t),
$$

for every $1 \leq k \leq n$. In other words, in terms of point processes this means

$$
\sum_{k=1}^{n} \delta_{\left|z_{k}\right|} \stackrel{d}{=} \sum_{k=1}^{n} \delta_{R_{k}}
$$

Theorem 1.2 follows from the approach of Kostlan [14] used in the Gaussian case $V(r)=$ $r^{2}$ (see also [12, Theorem 4.7.1] for a determinantal point processes point of view). Let us quickly recall the proof for convenience, which is elementary. 
Proof of Theorem 1.2. One starts from the Vandermonde determinant

$$
\prod_{1 \leq j<k \leq n}\left|z_{j}-z_{k}\right|=\operatorname{det}\left(\left(z_{j}^{k-1}\right)_{1 \leq j, k \leq n}\right)=\left|\sum_{\sigma \in S_{n}}(-1)^{\operatorname{sig}(\sigma)} \prod_{j=1}^{n} z_{j}^{\sigma(j)-1}\right| .
$$

Hence, the density 1.1 rewritten in polar coordinates $\left(z_{j}=r_{j} e^{i \theta_{j}}\right)$ is proportional to

$$
\left(r_{1}, \ldots, r_{n}, \theta_{1}, \ldots, \theta_{n}\right) \mapsto e^{-n \sum_{j=1}^{n} V\left(r_{j}\right)}\left|\sum_{\sigma \in S_{n}}(-1)^{\operatorname{sig}(\sigma)} \prod_{j=1}^{n} r_{j}^{\sigma(j)-1} e^{i(\sigma(j)-1) \theta_{j}}\right|^{2} \prod_{j=1}^{n} r_{j} .
$$

At this step, we observe that if $\sigma, \sigma^{\prime} \in S_{n}$ with $\sigma(k) \neq \sigma^{\prime}(k)$ for some $k$ then

$$
\int_{0}^{2 \pi}\left(\prod_{j=1}^{n} e^{i(\sigma(j)-1) \theta_{j}}\right) \overline{\left(\prod_{j=1}^{n} e^{i\left(\sigma^{\prime}(j)-1\right) \theta_{j}}\right)} d \theta_{k}=0 .
$$

Consequently, we obtain

$$
\int_{[0,2 \pi]^{n}} \prod_{1 \leq j<k \leq n}\left|z_{j}-z_{k}\right|^{2} d \theta_{1} \cdots d \theta_{n}=(2 \pi)^{n} \sum_{\sigma \in S_{n}} \prod_{j=1}^{n} r_{j}^{2(\sigma(j)-1)}=(2 \pi)^{n} \operatorname{per}\left[r_{j}^{2(k-1)}\right]_{1 \leq j, k \leq n}
$$

("per" stands for "permanent") and thus, the density of the moduli is proportional to

$$
e^{-n \sum_{j=1}^{n} V\left(r_{j}\right)} \operatorname{per}\left[r_{j}^{2(k-1)}\right]_{1 \leq j, k \leq n} \prod_{j=1}^{n} r_{j}=\operatorname{per}\left[r_{j}^{2 k-1} e^{-n V\left(r_{j}\right)}\right]_{1 \leq j, k \leq n}=\operatorname{per}\left[f_{k}\left(r_{j}\right)\right]_{1 \leq j, k \leq n} .
$$

Up to a factor $1 / n$ !, this is the density of $R_{\sigma(1)}, \ldots, R_{\sigma(n)}$ where $R_{1}, \ldots, R_{n}$ are independent random variables with $R_{k}$ of density proportional to $t \mapsto t^{2 k-1} e^{-n V(t)} \mathbf{1}_{[0, \infty)}(t)$ for every $1 \leq k \leq n$, and where $\sigma$ is a random uniform element of $S_{n}$, independent of $R_{1}, \ldots, R_{n}$.

Beware that the phases are not independent in Theorem 1.2. However, since the gas is rotationally invariant, we may deduce that the equilibrium measure $\mu_{Q}$ exists, is rotationally invariant, and we may compute its radial part using the law of the moduli.

If $V(r)=r^{2}$ then $\sqrt{n} R_{k}$ is $\chi$-distributed with $2 k$ degree of freedom. Theorem 1.2 allows to reuse the strategy behind the work of Rider [18] in the case where $V$ is a power. The following theorem is proved in Section 2, The case $\alpha=2$ is the one of Rider [18].

Theorem 1.3 (Power case). Let $|z|_{(1)}=\max _{1 \leq k \leq n}\left|z_{k}\right|$ be as in (1.2), with $\beta=2$. Suppose that $V(t)=t^{\alpha}$ for any $t \geq 0$, for some $\alpha \geq 1$. Set $c_{n}:=\log (n)-2 \log \log (n)-\log (2 \pi)$ and

$$
a_{n}:=2\left(\frac{\alpha}{2}\right)^{1 / \alpha+1 / 2} \sqrt{n c_{n}} \quad \text { and } \quad b_{n}:=\left(\frac{2}{\alpha}\right)^{1 / \alpha}\left(1+\frac{1}{2} \sqrt{\frac{2}{\alpha} \frac{c_{n}}{n}}\right) .
$$

Then $\left(a_{n}\left(|z|_{(1)}-b_{n}\right)\right)_{n \geq 1}$ converges in distribution as $n \rightarrow \infty$ to the standard Gumbel law:

$$
\forall x \in \mathbb{R}, \quad \lim _{n \rightarrow \infty} \mathbb{P}\left(a_{n}\left(|z|_{(1)}-b_{n}\right) \leq x\right)=e^{-e^{-x}} .
$$

In particular $\left(|z|_{(1)}\right)_{n \geq 1}$ converges in probability to $(2 / \alpha)^{1 / \alpha}$ as $n \rightarrow \infty$, in other words

$$
\forall \varepsilon>0, \quad \lim _{n \rightarrow \infty} \mathbb{P}\left(\left.|| z\right|_{(1)}-(2 / \alpha)^{1 / \alpha} \mid>\varepsilon\right)=0 .
$$

Our next result below, which is proved in Section 3, goes beyond the power case considered in Theorem 1.3 (which corresponds formally to the special choice $V(t)=t^{\alpha}$ ).

Theorem 1.4 (Beyond the power case). Let $|z|_{(1)}=\max _{1 \leq k \leq n}\left|z_{k}\right|$ be as in (1.2), with $\beta=2$. Additionally, let us assume the following properties on the potential $V$ :

- (A1) $V$ is strictly convex: there exists a $>0$ such that $V^{\prime \prime}(u) \geq a, \forall u \geq 0$;

- (A2) For each $x \in[0,2]$, there exists a unique $t_{x}$ such that $t_{x} V^{\prime}\left(t_{x}\right)=2-x$. 
Let $t_{0}$ be the unique solution to the equation: $t_{0} V^{\prime}\left(t_{0}\right)=2$. Let us define the sequences

$$
a_{n}:=\frac{\sqrt{n c_{n}}}{C_{0}} \quad \text { and } \quad b_{n}:=t_{0}+C_{0} \sqrt{\frac{c_{n}}{n}}
$$

where $C_{0}:=1 / \sqrt{\left|2 / t_{0}^{2}+V^{\prime \prime}\left(t_{0}\right)\right|^{3 / 2} t_{0} / 2}$ and

$$
c_{n}:=2 \log \left(\frac{\sqrt{n /(2 \pi)}}{\log (n)}\right)=\log (n)-2 \log \log (n)-\log (2 \pi) .
$$

Then $\left(a_{n}\left(|z|_{(1)}-b_{n}\right)\right)_{n \geq 1}$ converges in distribution as $n \rightarrow \infty$ to the standard Gumbel law:

$$
\forall x \in \mathbb{R}, \quad \lim _{n \rightarrow \infty} \mathbb{P}\left(a_{n}\left(|z|_{(1)}-b_{n}\right) \leq x\right)=e^{-e^{-x}} .
$$

In particular, $\left(|z|_{(1)}\right)_{n \geq 1}$ converges in probability to $t_{0}$ as $n \rightarrow \infty$, in other words,

$$
\forall \varepsilon>0, \quad \lim _{n \rightarrow \infty} \mathbb{P}\left(\left.|| z\right|_{(1)}-t_{0} \mid>\varepsilon\right)=0 .
$$

Weakly confining potentials and heavy tails. If the confining potential is not strong enough, the asymptotic fluctuation of $|z|_{(1)}$ is no longer Gumbel. For instance, in the case where $V(t)=c \log \left(1+t^{2}\right)$ for $c>1$, we get from Theorem 1.2 that $R_{k}$ has density proportional to $t \mapsto t^{2 k-1} /\left(1+t^{2}\right)^{c n} \mathbf{1}_{[0, \infty)}(t)$. In this case, the muduli of the particles are heavy tailed, and the equilibrium measure $\mu_{Q}$ exists and is heavy tailed. One may wonder if the largest particle in modulus $|z|_{(1)}$ has Fréchet type fluctuations as $n \rightarrow \infty$. Recall that a random variable $H$ follows the Fréchet law of parameter $\alpha>0$ iif $\mathbb{P}(H \leq t)=\exp \left(-t^{-\alpha}\right)$ for any $t>0$. In particular, $\mathbb{P}(H \leq t) \approx 1-t^{-\alpha}$ as $t \gg 1$. At least formally, if one takes

$$
V(t)=V_{n}(t)=+\infty \mathbf{1}_{0<t<1}+(2(1+1 / n)) \log (t) \mathbf{1}_{t \geq 1}
$$

then $t^{2 k-1} e^{-n V(t)}=t^{-2(n+1-k)-1} \mathbf{1}_{t \geq 1}$ and thus for every $t \geq 1$

$$
\mathbb{P}\left(\max _{1 \leq k \leq n} R_{k} \leq t\right)=\prod_{k=1}^{n} \mathbb{P}\left(R_{k} \leq t\right)=\prod_{k=1}^{n}\left(1-t^{-2(n+1-k)}\right)=\prod_{k=1}^{n}\left(1-t^{-2 k}\right)
$$

which gives

$$
\log \mathbb{P}\left(\max _{1 \leq k \leq n} R_{k} \leq t\right)=\sum_{k=1}^{n} \log \left(1-t^{-2 k}\right) \stackrel{t \gg 1}{\approx}-\sum_{k=1}^{n} t^{-2 k} \stackrel{n \gg 1}{\approx}-1 /\left(t^{2}-1\right)
$$

and therefore

$$
\mathbb{P}\left(\max _{1 \leq k \leq n} R_{k} \leq t\right) \stackrel{t, n \gg 1}{\approx} e^{-1 /\left(t^{2}-1\right)} \approx 1-1 /\left(t^{2}-1\right) \approx 1-1 / t^{2}=\mathbb{P}\left(R_{n} \leq t\right) .
$$

The phenomenon is that a sum behaves asymptically like its largest term, which implies for the particles that the largest in modulus behaves like the one of largest index.

Comments and open problems. The edge universality remains untouched for general $\beta$-ensembles on the complex plane and for the eigenvalues of general random matrices with i.i.d. entries (spectral radius). The complex Ginibre ensemble is exactly solvable and belongs to both categories. The method used in the proof of Theorem 1.2 is maybe still usable when $\beta$ is an even integer. One may also use it for gases in $\mathbb{R}^{d}, d>3$, with density proportional to $\prod_{j=1}^{n} e^{-n Q\left(z_{j}\right)} \prod_{1 \leq j<k \leq n}\left\|z_{j}-z_{k}\right\|_{2}^{2}$, in order to obtain the law of the norms of the particles (by integrating non radial variables, possibly via a sort of Wick formula on spheres). A study of the bulk universality beyond logarithmic repulsion is considered in [9]. On the other side, it is conjectured that the spectral radius of square random matrices with i.i.d. centered entries of variance $1 / n$ and finite fourth moment has also a Gumbel type asymptotic fluctuations as $n \rightarrow \infty$, making the Ginibre case universal again.

One may ask about the universality of refined aspects of the complex Ginibre ensemble, such as the order statistics of the moduli of the eigenvalues, studied by Rider in [19]. One 
may ask about the fluctuation of the smallest particle in modulus $|z|_{(n)}$ instead on the largest $|z|_{(1)}$. For a general radially symmetric $Q$, the equilibrium measure $\mu_{Q}$ is radially symmetric but might be supported by more than one ring (lack of radial connectivity). One may then study the fluctuation at each edge (inner and outer) of these rings. Another natural question is to ask about the fluctuation at the edge in the single ring theorem [10].

Acknowledgments. This note benefited from the comments of two anonymous reviewers.

\section{Proof of Theorem 1.3}

Proof of Theorem 1.3. From Theorem [1.2, $n|z|_{(k)}^{\alpha}$ has density proportional to $t^{\frac{2 k}{\alpha}-1} e^{-t}$ which is $\Gamma(2 k / \alpha, 1)$, for every $1 \leq k \leq n$. This gives the identity in distribution

$$
n|z|_{(k)}^{\alpha} \stackrel{d}{=} X_{1}+\cdots+X_{k}
$$

where $X_{1}, \ldots, X_{k}$ are i.i.d. of law $\Gamma(2 / \alpha, 1)$ (mean and variance both equal to $2 / \alpha$ ). Thus, for any deterministic sequences $\left(A_{n}\right)$ and $\left(B_{n}\right)$ in $(0, \infty)$, and every $x \in \mathbb{R}$,

$$
\mathbb{P}\left(A_{n}\left(|z|_{(1)}^{\alpha}-B_{n}\right) \leq x\right)=\prod_{k=1}^{n} \mathbb{P}\left(\frac{X_{1}+\cdots+X_{k}}{n} \leq \frac{x}{A_{n}}+B_{n}\right) .
$$

Let $c_{n}:=2 \log \left(\frac{\sqrt{n /(2 \pi)}}{\log (n)}\right)=\log (n)-2 \log \log (n)-\log (2 \pi)$. Lemma 2.1 gives now that for

$$
A_{n}=\sqrt{\frac{\alpha}{2} n c_{n}} \quad \text { and } \quad B_{n}=\frac{2}{\alpha}+\sqrt{\frac{2}{\alpha} \frac{c_{n}}{n}}
$$

and for every $x \in \mathbb{R}$,

$$
\lim _{n \rightarrow \infty} \prod_{k=1}^{n} \mathbb{P}\left(\frac{X_{1}+\cdots+X_{k}}{n} \leq \frac{x}{A_{n}}+B_{n}\right)=e^{-e^{-x}} .
$$

It remains finally to use Lemma 2.2 with $f(x)=x^{1 / \alpha}$ and $Z_{n}=|z|_{(1)}=\max _{1 \leq k \leq n}\left|z_{k}\right|$ (one has also to use the Slutsky lemma to obtain a bit nicer shift parameter $b_{n}$ ).

Lemma 2.1 (Special products). If $\left(X_{n}\right)_{n \geq 1}$ are i.i.d. real random variables such that

(i) $\mathbb{E}\left(e^{\theta\left|X_{1}\right|}\right)<\infty$ for some $\theta>0$;

(ii) $\mathbb{P}\left(X_{1} \geq-a\right)=1$ for some $a>0$;

(iii) $m=\mathbb{E}\left(X_{1}\right)>0$ and $\sigma^{2}=\operatorname{Var}\left(X_{1}\right)>0$;

then

$$
\forall x \in \mathbb{R}, \quad \lim _{n \rightarrow \infty} \prod_{k=1}^{n} \mathbb{P}\left(\frac{X_{1}+\cdots+X_{k}}{n} \leq \frac{x}{A_{n}}+B_{n}\right)=e^{-e^{-x}}
$$

where $c_{n}:=2 \log \left(\frac{\sqrt{n /(2 \pi)}}{\log (n)}\right)=\log (n)-2 \log \log (n)-\log (2 \pi)$ and

$$
A_{n}:=\frac{\sqrt{n c_{n}}}{\sigma} \quad \text { and } \quad B_{n}:=m+\sigma \sqrt{\frac{c_{n}}{n}} .
$$

Note that in the case were $X_{k} \sim \Gamma\left(a_{k}, \lambda\right)$ for every $k$ then every probability in the product can be expressed in terms of the incomplete Gamma function.

Proof. We adapt the argument used by Rider [18] in the case of exponential random variables. The driving intuitive idea is based on the Law of Large Numbers and the Central Limit Theorem, which indicate that the probabilities under the product are either asymptotically 1 or identical to a Gaussian deviation probability, leading to the maximum of independent Gaussians, which is known to be Gumbel. Namely, let $\left(K_{n}\right)_{n \geq 1}$ and $\left(\delta_{n}\right)_{n \geq 1}$ 
be deterministic sequences such that $K_{n}=\mathcal{O}(\log (n))$ and $\delta_{n}=\mathcal{O}(\sqrt{\log (n)} / \sqrt{n})$, and let $f_{n}$ be the increasing function in both $n$ and $x$ given by

$$
f_{n}(x):=\sqrt{2 \log \left(\frac{e^{x} \sqrt{n /(2 \pi)}}{\log (n)}\right)}=\sqrt{2 x+c_{n}} .
$$

The choices of $A_{n}$ and $B_{n}$ come from the following equivalence as $n \rightarrow \infty$

$$
\sigma \frac{f_{n}(x)}{\sqrt{n}}+m=\sigma \sqrt{\frac{2 x+c_{n}}{n}}+m \sim \sigma \sqrt{\frac{c_{n}}{n}}\left(1+\frac{x}{c_{n}}\right)+m=: \frac{x}{A_{n}}+B_{n} .
$$

From Theorem 1.2 we have

$$
\mathbb{P}\left(|z|_{(1)} \leq \sigma \frac{f_{n}(x)}{\sqrt{n}}+m\right)=\prod_{k=1}^{n} \mathbb{P}\left(\frac{1}{n} \sum_{i=1}^{n-k} X_{i} \leq \sigma \frac{f_{n}(x)}{\sqrt{n}}+m\right) .
$$

We will show that the product over $1 \leq k \leq \delta_{n} n$ terms goes to $e^{-e^{-x}}$ as $n \rightarrow \infty$ while the product over $\delta_{n} n \leq k \leq n-1$ tends to 1 as $n \rightarrow \infty$. Namely, denoting

$$
Y_{i}:=\frac{X_{i}-m}{\sigma} \text { and } g_{n, k}(x):=\sqrt{\frac{n}{n-k}}\left(f_{n}(x)+\frac{m}{\sigma} \frac{k}{\sqrt{n}}\right)
$$

we get,

$$
\left\{\frac{1}{n} \sum_{i=1}^{n-k} X_{i} \leq \sigma \frac{f_{n}(x)}{\sqrt{n}}+m\right\}=\left\{\frac{1}{\sqrt{n-k}} \sum_{i=1}^{n-k} Y_{i} \leq g_{n, k}(x)\right\} .
$$

Let now $L$ be chosen large enough. We can assume that $n$ is large enough so that $K_{n} \gg$ $\sup _{|x| \leq L}\left|f_{n}(x)\right|$. Using quantitative Central Limit Theorem (Edgeworth expansion [3]) and assuming $|x| \leq L$, one has that for a given $k \in\left[n \delta_{n}, n\right]$

$$
\begin{aligned}
& \log \mathbb{P}\left(\frac{1}{\sqrt{n-k}} \sum_{i=1}^{n-k} Y_{i} \leq g_{n, k}(x)\right) \\
&=\log \int_{-K_{n}}^{g_{n, k}(x)} \frac{e^{-t^{2} / 2}}{\sqrt{2 \pi}} d t+\mathcal{O}\left(\frac{1}{\sqrt{n}} \sup _{|x| \leq L}\left|f_{n}^{2}(x)\right| e^{-f_{n}^{2}(x) / 2}+1 / n+K_{n} / n^{3 / 2}\right) \\
&+\mathcal{O}\left(e^{-c K_{n}^{2}}\right) .
\end{aligned}
$$

The last term stems from Hoeffding's concentration inequality to bound from above the probability that $\frac{1}{\sqrt{n-k}} \sum_{i=1}^{n-k} Y_{i} \leq-K_{n}$. Indeed, first one may use (ii) to get

$$
\mathbb{P}\left(\frac{1}{\sqrt{n-k}} \sum_{i=1}^{n-k} Y_{i} \leq-K_{n}\right) \leq \mathbb{P}\left(\frac{1}{\sqrt{n-k}} \sum_{i=1}^{n-k} Y_{i} \mathbf{1}_{\left|X_{i}-m\right| \leq m+a} \leq-K_{n}\right) .
$$

Next, when $k$ runs from 1 to $n \delta_{n}=\mathcal{O}(\sqrt{n \log (n)})$ and when $n \gg 1$ and $n \delta_{n} \gg 1$, one may see $g_{n, k}$ as an interpolation between $f_{n}(x)$ and $+\infty$. By copying the arguments of [18, which essentially amounts to the Riemann sum approximation of an integral, we obtain

$$
\begin{aligned}
& \sum_{1 \leq k<n \delta_{n}} \log \mathbb{P}\left(\frac{1}{\sqrt{n-k}} \sum_{i=1}^{n-k} Y_{i} \leq g_{n, k}(x)\right) \\
&=\sqrt{n} \int_{f_{n}(x)}^{\infty} \log \left(\int_{-\infty}^{t} e^{-s^{2} / 2} \frac{d s}{\sqrt{2 \pi}}\right) d t \\
&+\mathcal{O}\left(\frac{\log (n)}{\sqrt{n}}+\sqrt{\log (n)} \sup _{|x| \leq L} f_{n}^{2}(x) e^{-f_{n}^{2}(x) / 2}\right) .
\end{aligned}
$$


With our choice of $f_{n}(x)$, we get [18, eq. (15)] that uniformly on compact sets in $x$

$$
\lim _{n \rightarrow \infty} \log \prod_{1 \leq k<n \delta_{n}} \mathbb{P}\left(\frac{1}{n} \sum_{i=1}^{n-k} X_{i} \leq \sigma \frac{f_{n}(x)}{\sqrt{n}}+m\right)=-e^{-x} .
$$

We now examine the contribution of the remaining terms:

$$
\prod_{k=n \delta_{n}}^{n-1} \mathbb{P}\left(\frac{1}{n} \sum_{i=1}^{n-k} X_{i} \leq \sigma \frac{f_{n}(x)}{\sqrt{n}}+m\right)
$$

As the product is never larger than 1, one only needs to get a lower bound. Now, by (i),

$$
\begin{aligned}
\prod_{k=n \delta_{n}}^{n-1} \mathbb{P}\left(\frac{1}{n} \sum_{i=1}^{n-k} X_{i} \leq \sigma \frac{f_{n}(x)}{\sqrt{n}}+m\right) & \geq \prod_{k=n \delta_{n}}^{n-1}\left(1-\mathbb{P}\left(\sum_{i=1}^{n-k} X_{i} \geq \sigma \sqrt{n} f_{n}(x)+n m\right)\right) \\
& \geq \prod_{k=n \delta_{n}}^{n-1}\left(1-e^{-\theta \sigma \sqrt{n} f_{n}(x)-\theta n m} \mathbb{E}\left(e^{\theta X_{1}}\right)^{n-k}\right)
\end{aligned}
$$

For any $k$ such that $(n-k) / n<c<1$ it is not difficult to see that

$$
1-e^{-\theta \sigma \sqrt{n} f_{n}(x)-\theta n m_{m}} \mathbb{E}\left(e^{\theta X_{1}}\right)^{n-k} \geq\left(1-e^{-C n}\right),
$$

where the constant $C>0$ depends on $c<1$. All the difficulty lies in the regime where $n-k \sim n$. In this case we can use the moderate deviation result of [16, Th. 5.23 p. 189] to get that for any $n \delta_{n} \leq k \leq n(1-\epsilon)$

$$
\mathbb{P}\left(\sum_{i=1}^{n-k} X_{i} \geq \sigma \sqrt{n} f_{n}(x)+n m\right) \leq e^{-C_{0} \log (n-k)} .
$$

Actually we can refine the estimate when $n^{3 / 4} \leq k \leq(1-\epsilon) n$

$$
\mathbb{P}\left(\sum_{i=1}^{n-k} X_{i} \geq \sigma \sqrt{n} f_{n}(x)+n m\right) \leq e^{-C_{0} n^{1 / 4}} .
$$

Combining the whole, we deduce that

$$
\begin{aligned}
\prod_{k=n \delta_{n}}^{n-1} \mathbb{P}\left(\frac{1}{n} \sum_{i=1}^{n-k} X_{i} \leq \sigma \frac{f_{n}(x)}{\sqrt{n}}\right. & +m) \\
& \geq\left(1-e^{-c n}\right)^{n(1-1 / c)}\left(1-e^{-C_{0} n^{1 / 4}}\right)^{n c}\left(1-e^{-C_{0} \log (n-k)}\right)^{n \delta_{n}},
\end{aligned}
$$

which obviously goes to 1 as $n \rightarrow \infty$.

Lemma 2.2 (An instance of the delta method). Let $\left(Z_{n}\right)$ be a sequence of random variables on $(0, \infty)$ and let $f:(0, \infty) \rightarrow \mathbb{R}$ be a $\mathcal{C}^{1}$ function such as $f(x)=x^{\rho}$ with $\rho>0$. If there exist deterministic sequences $\left(A_{n}\right)$ and $\left(B_{n}\right)$ in $(0, \infty)$ such that

(i) $A_{n}\left(Z_{n}-B_{n}\right)$ converges in law as $n \rightarrow \infty$ to some probability distribution $P$;

(ii) $A_{n} \rightarrow \infty$;

(iii) $B_{n} \rightarrow B>0$

(iv) $f^{\prime}(B) \neq 0$;

then, denoting $a_{n}=A_{n} / f^{\prime}(B)$ and $b_{n}=f\left(B_{n}\right)$, the sequence $a_{n}\left(f\left(Z_{n}\right)-b_{n}\right)$ converges also in law as $n \rightarrow \infty$ to the same probability distribution $P$.

Proof. We may use a Taylor formula (or intermediate value theorem) to get

$$
f\left(Z_{n}\right)-f\left(B_{n}\right)=f^{\prime}\left(W_{n}\right)\left(Z_{n}-B_{n}\right)
$$

where $W_{n}$ is a random variable lying between $B_{n}$ and $Z_{n}$. Thanks to (i), (ii), and the Slutsky lemma, we have $Z_{n}-B_{n} \rightarrow 0$ in law, and thus in probability because the limit 
is deterministic. Therefore $W_{n}-B_{n} \rightarrow 0$ in probability, and thus, by (iii), $W_{n} \rightarrow B$ in probability. Since $f^{\prime}$ is continuous at point $B>0$, the continuous mapping theorem gives that $f^{\prime}\left(W_{n}\right) \rightarrow f^{\prime}(B)$ in probability, and thus, by (iv), $f^{\prime}\left(W_{n}\right) / f^{\prime}(B) \rightarrow 1$ in probability. Now it remains to use (i) and the Slutsky lemma to obtain

$$
a_{n}\left(f\left(Z_{n}\right)-b_{n}\right)=\frac{A_{n}}{f^{\prime}(B)}\left(f\left(Z_{n}\right)-f\left(B_{n}\right)\right)=\frac{f^{\prime}\left(W_{n}\right)}{f^{\prime}(B)} A_{n}\left(Z_{n}-B_{n}\right) \stackrel{d}{\longrightarrow} P .
$$

\section{Proof of Theorem 1.4}

This section is devoted to the proof of Theorem 1.4. We thus now consider the case where the potential $V$ is rather general satisfying the following assumptions:

- (A1) $V$ is strictly convex: there exists $a>0$ such that $V^{\prime \prime}(u) \geq a, \forall u \geq 0$.

- (A2) For each $x \in[0,2]$, there exists a unique $t_{x}$ such that $t_{x} V^{\prime}\left(t_{x}\right)=2-x$.

Theorem 1.4 follows from the Lemma 3.1 below. Namely, Lemma 3.1 gives

$$
\forall u \in \mathbb{R}, \quad \lim _{n \rightarrow \infty} \mathbb{P}\left(\sqrt{n}\left(|z|_{(1)}-t_{0}\right) \leq f_{n}(u)\right)=e^{-e^{-u}}
$$

where

$$
f_{n}(u)=\sqrt{2 u+c_{n}}=\sqrt{c_{n}} \sqrt{1+2 u / c_{n}}=\sqrt{c_{n}}\left(1+u / c_{n}+o\left(c_{n}\right)\right),
$$

which leads to

$$
\forall u \in \mathbb{R}, \quad \lim _{n \rightarrow \infty} \mathbb{P}\left(a_{n}\left(|z|_{(1)}-b_{n}\right) \leq u\right)=e^{-e^{-u}}
$$

where

$$
a_{n}:=\frac{\sqrt{n c_{n}}}{C_{0}} \text { and } \quad b_{n}:=t_{0}+C_{0} \sqrt{\frac{c_{n}}{n}} .
$$

Lemma 3.1. Let $t_{0}$ be the unique solution to the equation : $t_{0} V^{\prime}\left(t_{0}\right)=2$. Set also

$$
C_{0}:=\frac{1}{\sqrt{\left|F^{\prime \prime}\left(t_{0}\right)\right|^{3 / 2} \frac{1}{2} t_{0}}} \text { and } f_{n}(u):=\sqrt{2 \log \left(\frac{e^{u} \sqrt{n /(2 \pi)}}{\log (n)}\right)}
$$

where $F^{\prime \prime}\left(t_{0}\right):=-2 / t_{0}^{2}-V^{\prime \prime}\left(t_{0}\right)$. Then for every $U_{0}>0$, uniformly in $u \in\left[-U_{0}, U_{0}\right]$,

$$
\lim _{n \rightarrow \infty} \mathbb{P}\left(|z|_{(1)} \leq t_{0}+C_{0} \frac{f_{n}(u)}{\sqrt{n}}\right)=e^{-e^{-u}} .
$$

Remark 3.2 (Relaxed assumption). Assumption (A1) can in principle be relaxed to the strict convexity of $V(t)-\frac{2 k-1}{n} \log (t)$ for all $1 \leq k \leq n$. The asymptotic expansion can then be performed unchanged provided that there exists $a>0$ so that for any such $k$

$$
\forall t>0, \quad V^{\prime \prime}(t)+\frac{2 k-1}{n} t^{-2}>a .
$$

Proof of Lemma 3.1. Recall that $\left(|z|_{(1)}, \ldots,|z|_{(n)}\right)$ is distributed as the order statistic $\left(X_{(1)}, \ldots, X_{(n)}\right)$ of $X_{1}, \ldots, X_{n}$ which are independent random variables with $X_{i}$ of density proportional to $t \mapsto t^{2 i-1} e^{-n V(t)}$. We shall first consider the value (if it exists) around which the random variables $X_{i}$ concentrate for a large $i$. Typically if $\lim _{n \rightarrow \infty}(i / n)=2-x$, we here show that $X_{i}$ concentrates around the deterministic number $t_{x}$. Indeed, if $f$ be a bounded continuous function $f: \mathbb{R} \rightarrow \mathbb{R}$, then we consider the asymptotic expansion of $\mathbb{E}\left(f\left(X_{i}\right)\right)$ when $i / n \rightarrow 2-x>0$ using the Laplace method. We have

where

$$
\mathbb{E}\left(f\left(X_{i}\right)\right)=\frac{1}{Z_{i}} \int_{0}^{\infty} e^{n F_{i}(t)} f(t) d t
$$

$$
Z_{i}:=\int_{0}^{\infty} t^{2 i-1} e^{-n V(t)} d t \quad \text { and } \quad F_{i}(t):=\frac{2 i-1}{n} \log (t)-V(t) .
$$


To determine critical points, one uses that

$$
F_{i}^{\prime}(t)=\frac{2 i-1}{n t}-V^{\prime}(t)
$$

By assumption (A2), there exists a unique solution $t_{(i)}$ to the equation $F_{i}^{\prime}(t)=0$ and by (A1) one has that $\left|t_{(i)}-t_{x}\right| \leq C_{0}(1 / n+|x-i / n|)$ for some constant $C_{0}$ (independent of $x, i, n)$. Also by (A1) this critical point is non degenerate as

$$
F_{i}^{\prime \prime}(t)=-\frac{2 i-1}{n t^{2}}-V^{\prime \prime}(t)<-a
$$

Note that this readily implies the Gaussian decay of the exponential term: for all $t \in \mathbb{R}$,

$$
F_{i}(t)-F_{i}\left(t_{i}\right) \leq-\frac{a}{2}\left(t-t_{i}\right)^{2}
$$

By a standard Laplace approximation, one then deduces that for any $x \in[0,2[$ and for any $i$ such that $i / n \rightarrow 2-x$ the following holds:

$$
\mathbb{E} f\left(X_{i}\right)=f\left(t_{i}\right)\left(1+\mathcal{O}\left(n^{-1 / 2}\right)\right)=f\left(t_{x}\right)(1+o(1)) .
$$

As a consequence, if $i$ is very large, that is those integers $i$ such that

$$
\frac{i}{n}=1-\epsilon_{n}, \quad \text { with } \epsilon_{n} \rightarrow 0 \text { as } n \rightarrow \infty,
$$

using that

$$
t_{i}-t_{0}=-\frac{-2}{\left|t_{0} F^{\prime \prime}\left(t_{0}\right)\right|}\left(\epsilon_{n}+\frac{1}{n}\right)+o\left(\epsilon_{n}\right)
$$

we conclude that the $X_{i}$ 's concentrate around $t_{0}$. The constant $t_{0}$ will play the role of $a_{n}$ in the previous analysis.

Let us now try to give the main ideas of the rest of the proof. Let $u>0$ be a given real number. Using Theorem 1.2, in order to determine the limiting distribution of $|z|_{(1)}$ we consider the following:

$$
\mathbb{P}\left(|z|_{(1)} \leq t_{0}+\frac{g_{n}(u)}{\sqrt{n}}\right)=\prod_{i=1}^{n} \mathbb{P}\left(X_{i} \leq t_{0}+\frac{g_{n}(u)}{\sqrt{n}}\right),
$$

where $g_{n}(x)$ is growing to infinity with $n$ and to be determined. Consider first

$$
\prod_{i=i_{0}}^{n} \mathbb{P}\left(X_{i} \leq t_{0}+\frac{g_{n}(u)}{\sqrt{n}}\right)
$$

for some $i_{0}$ that we choose as

$$
i_{0}:=n-c \sqrt{n \log (n)} .
$$

The value of the real $c>0$ will be fixed later. One can first observe that $i_{0} \geq \epsilon n$ for some $\epsilon>0$. For each $i \geq i_{0}$ one has that

$$
\begin{aligned}
& \mathbb{P}\left(X_{i} \leq t_{0}+\frac{g_{n}(u)}{\sqrt{n}}\right)=\frac{1}{Z_{i}} \int_{-\infty}^{t_{0}+n^{-1 / 2} g_{n}(u)} e^{n F_{i}(t)} d t \\
& =\frac{\left(F_{i}^{\prime \prime}\left(t_{i}\right) n\right)^{1 / 2}}{\sqrt{2 \pi} e^{n F_{i}\left(t_{i}\right)}\left(1+\mathcal{O}\left(n^{-3 / 4}\right)\right)} \int_{-\infty}^{t_{0}+n^{-1 / 2} g_{n}(u)} e^{n F_{i}(t)} d t \\
& =\frac{\left(F_{i}^{\prime \prime}\left(t_{i}\right) n\right)^{1 / 2}}{\sqrt{2 \pi}\left(1+\mathcal{O}\left(n^{-3 / 4}\right)\right)} \int_{-\infty}^{t_{i}+t_{0}-t_{i}+n^{-1 / 2} g_{n}(u)} e^{n\left(F_{i}(t)-F_{i}\left(t_{i}\right)\right)} d t \\
& =\frac{\left(F_{i}^{\prime \prime}\left(t_{i}\right)\right)^{1 / 2}}{\sqrt{2 \pi}\left(1+\mathcal{O}\left(n^{-3 / 4}\right)\right)} \int_{-\infty}^{\sqrt{n}\left(t_{0}-t_{i}\right)+g_{n}(u)} e^{n\left(F_{i}\left(t_{i}+\frac{s}{n^{1 / 2}}\right)-F_{i}\left(t_{i}\right)\right)} d s .
\end{aligned}
$$


Hereabove we have used the asymptotic expansion of $Z_{i}$ using the Laplace method, and the fact that $i \geq \epsilon n$ for the error control, and in the last line we made the change of variables $t=t_{i}+s / \sqrt{n}$.

Assume now that $g_{n}(u) \ll n^{1 / 2}$. Because $t_{i} \leq t_{0}$, the integral in (3.1) is of order $\mathcal{O}(1)$. We need to refine this rough order. To that aim, we need to control the variation of $F_{i}$ (and the Gaussian decay) in a bounded neighborhood of $t_{i}$. Let $\delta>0$ be given (small), that we fix hereafter. Let us first examine the decay of $F_{i}$ "far" from $t_{i}$. Using the upper bound $F_{i}(t)-F_{i}\left(t_{i}\right) \leq-a\left(t-t_{i}\right)^{2} / 2$ forall $t$, one can see that only a neighborhood of $t_{i}$ of width $n^{-1 / 2}$ can contribute to the integral. We then turn to a neighborhood of $t_{i}$.

$$
\left|n F_{i}\left(t_{i}+s\right)-n F_{i}\left(t_{i}\right)-n F_{i}^{\prime \prime}\left(t_{i}\right) \frac{s^{2}}{2}\right| \leq n \sup _{\left|t-t_{i}\right| \leq \delta}\left|F_{I}^{\prime \prime \prime}(t)\right| \frac{s^{3}}{3 !} \leq n \delta \sup _{\left|t-t_{i}\right| \leq \delta}\left|F_{I}^{\prime \prime \prime}(t)\right| \frac{s^{2}}{6}
$$

One can choose $\delta$ small enough so that for any $s,|s|<\delta$

$$
\left|n F_{i}\left(t_{i}+s\right)-n F_{i}\left(t_{i}\right)-n F_{i}^{\prime \prime}\left(t_{i}\right) \frac{s^{2}}{2}\right| \leq n F_{i}^{\prime \prime}\left(t_{i}\right) \frac{s^{2}}{4} .
$$

Thus using the change of variables $t:=t_{i}+s / n^{1 / 2}$ and by a straightforward Taylor expansion, we deduce that for all $i \geq i_{0}$,

$$
\begin{aligned}
\left|\int_{|s| \leq \delta \sqrt{n}} e^{n\left(F_{i}\left(t_{i}+\frac{s}{n^{1 / 2}}\right)-F_{i}\left(t_{i}\right)\right)} d s-\int_{|s| \leq \delta \sqrt{n}} e^{-F_{i}^{\prime \prime}\left(t_{i}\right)^{2} s^{2} / 2} d s\right| \\
\quad \leq \sup _{i \geq i_{0}} \int_{|s| \leq \delta \sqrt{n}} n^{-1 / 2} \sup _{z \in B\left(t_{0}, \delta\right)}\left|F_{i}^{\prime \prime \prime}(z)\right| \frac{s^{3}}{6} e^{-C_{0} s^{2}} \leq C_{1} n^{-1 / 2} .
\end{aligned}
$$

The above estimate on the error $\mathcal{O}\left(n^{-1 / 2}\right)$ is not precise enough as, later, one will sum this error over $n^{1 / 2} \sqrt{\log (n)}$ integers $i \geq i_{0}$. We need to refine the above estimate.

Lemma 3.3 (Exponential expansion). If $h: \mathbb{R} \rightarrow \mathbb{R}$ is $\mathcal{C}^{4}$ function such that $h^{\prime}(0)=0$ and $h^{\prime \prime}(0)<0$ then there exists $\delta>0$ such that for all $|t| \leq \delta$,

$$
e^{h(t)-h(0)}-e^{\frac{h^{\prime \prime}(0) t^{2}}{2}}=\frac{h^{(3)}(0)}{6} t^{3} e^{\frac{h^{\prime \prime}(0) t^{2}}{2}}+\mathcal{O}(P(t)) e^{\frac{h^{\prime \prime}(0) t^{2}}{4}},
$$

where $P(t)=C_{1} t^{4}+C_{2} t^{6}$ for some constants $C_{1}>0$ and $C_{2}>0$.

Proof of Lemma 3.3. First, one can write

$$
h(t)-h(0)=\int_{0}^{t} \int_{0}^{s} h^{\prime \prime}(u) d u d s=t^{2} \int_{0}^{1} s d s \int_{0}^{1} h^{\prime \prime}(s u t) d u .
$$

From this, one deduces that

$$
e^{h(t)-h(0)}-e^{\frac{h^{\prime \prime}(0) t^{2}}{2}}=e^{\frac{h^{\prime \prime}(0) t^{2}}{2}}\left(e^{t^{2} \int_{0}^{1} s d s \int_{0}^{1}\left(h^{\prime \prime}(s u t)-h^{\prime \prime}(0)\right) d u}-1\right) .
$$

Set now $G(t):=t^{2} \int_{0}^{1} s d s \int_{0}^{1} d u\left(h^{\prime \prime}(s u t)-h^{\prime \prime}(0)\right)$. Then again

$$
G(t)=t^{3} \int_{0}^{1} s^{2} d s \int_{0}^{1} u d u \int_{0}^{1} h^{(3)}(s u t x) d x .
$$

Using that

$$
e^{X}-1=X+X^{2} \int_{0}^{1} t d t \int_{0}^{1} d u e^{X t u}
$$


one then deduces that

$$
\begin{aligned}
\frac{e^{h(t)-h(0)-e^{\frac{h^{\prime \prime}(0) t^{2}}{2}}}}{e^{\frac{h^{\prime \prime}(0) t^{2}}{2}}}= & e^{G(t)}-1 \\
= & G(t)+G(t)^{2} \int_{0}^{1} v d v \int_{0}^{1} d u e^{G(t) v u} x \\
= & \frac{t^{3}}{6} h^{(3)}(0)+t^{4} \int_{0}^{1} s^{3} d s \int_{0}^{1} u^{2} d u \int_{0}^{1} x d x \int_{0}^{1} h^{(4)}(\text { sut } x v) d v \\
& +G(t)^{2} \int_{0}^{1} v d v \int_{0}^{1} d u e^{G(t) v u} .
\end{aligned}
$$

Thus we get that, every $\delta>0$ and for all $t$ such that $|t| \leq \delta$,

$$
\left|e^{h(t)-h(0)}-e^{\frac{h^{\prime \prime}(0) t^{2}}{2}}-e^{\frac{h^{\prime \prime}(0) t^{2}}{2}} \frac{t^{3}}{6} h^{(3)}(0)\right| \leq e^{\frac{h^{\prime \prime}(0) t^{2}}{2}}\left(t^{4} C_{1} \sup _{|t| \leq \delta}\left|h^{(4)}(t)\right|+C_{2} t^{6} e^{G(t) \vee 0}\right) .
$$

As $|G(t)| \leq C_{3} t^{3}$ one then deduces that for $\delta$ small enough and any $|t| \leq \delta$,

$$
e^{\frac{h^{\prime \prime}(0) t^{2}}{2}+G(t)} \leq e^{\frac{h^{\prime \prime}(0) t^{2}}{4}}
$$

This yields the desired estimate.

Back to the proof of Lemma 3.1, thanks to Lemma 3.3, (3.2) can be improved to

$$
\begin{aligned}
\mid \int_{|s| \leq \delta \sqrt{n}} e^{n\left(F_{i}\left(t_{i}+\frac{s}{n^{1 / 2}}\right)-F_{i}\left(t_{i}\right)\right)} d s & -\int_{|s| \leq \delta \sqrt{n}} e^{-F_{i}^{\prime \prime}\left(t_{i}\right)^{2} \frac{s^{2}}{2}} d s \\
-\int_{|s| \leq \delta \sqrt{n}} e^{-F_{i}^{\prime \prime}\left(t_{i}\right)^{2} \frac{s^{2}}{2}} F_{i}^{(3)}\left(t_{i}\right) \frac{s^{3}}{6 n^{1 / 2}} d s \mid & \leq \int_{|s| \leq \delta \sqrt{n}} \frac{C_{1} s^{4}}{n}+\frac{C_{2} s^{6}}{n^{2}} e^{-C_{0} s^{2}} \leq C_{3} n^{-1} .
\end{aligned}
$$

Note first that the above estimates hold uniformy in $i \geq i_{0}$ provided that $i_{0} / n \rightarrow 1$ as $n$ goes to infinity. In particular the constants $C_{1}, C_{2}, C_{3}$ do not depend on $i \geq i_{0}$. Note also that the same estimate holds if one multiplies the integrand by the indicator function $\mathbf{1}_{s \leq \sqrt{n}\left(t_{0}-t_{i}\right)+g_{n}(u)}$. Let now $h_{n}<\delta \sqrt{n}$ be such that $h_{n} \rightarrow \infty$ as $n$ grows to infinity. Then, using the fact that the third derivative $F_{i}^{\prime \prime \prime}\left(t_{i}\right)$ is bounded uniformly in $i$ when $n-i \leq c \sqrt{n \log (n)}$, we deduce that

$$
\int_{\left|t-t_{i}\right| \leq \delta, s \leq h_{n}} e^{-F_{i}^{\prime \prime}\left(t_{i}\right)^{2} s^{2} / 2} F_{i}^{(3)}\left(t_{i}\right) \frac{s^{3}}{6 n^{1 / 2}} d s \leq C n^{-1 / 2} h_{n}^{2} e^{-h_{n}^{2}}
$$

Indeed the integral can be explicitly computed. Lastly one has that there exists a constant $C_{4}$ such that for any $i \geq i_{0}$

$$
\left|\int_{t<t_{i}-\delta} e^{n\left(F_{i}\left(t_{i}+\frac{s}{n^{1 / 2}}\right)-F_{i}\left(t_{i}\right)\right)} d s\right| \leq C_{4} e^{-n a \delta^{2} / 2} \text { and }\left|\int_{t<t_{i}-\delta} e^{n F_{i}^{\prime \prime}\left(t_{i}\right) s^{2} / 2} d s\right| \leq C_{4} e^{-n a \delta^{2} / 2} .
$$

Combining the whole yields that up to an error term (of order $n^{-1 / 2} h_{n}^{2} e^{-h_{n}^{2}}$ ) one can replace the exponential term by the Gaussian one obtained by the Taylor expansion. 
Let $L$ be some large positive real number. Here we assume that $|u| \leq L$ and prove uniform convergence on compact sets of the c.d.f.: one has that

$$
\begin{aligned}
\log & \prod_{i=i_{0}}^{n} \mathbb{P}\left(X_{i} \leq t_{0}+\frac{g_{n}(u)}{\sqrt{n}}\right) \\
& =\sum_{i \geq i_{0}}\left\{\log \left[\int_{-\infty}^{g_{n}(u)+\sqrt{n}\left(t_{0}-t_{i}\right)} \frac{\sqrt{F_{i}^{\prime \prime}\left(t_{i}\right)}}{\sqrt{2 \pi}} e^{-F_{i}^{\prime \prime}\left(t_{i}\right) s^{2} / 2} d s+\mathcal{O}\left(n^{-1 / 2} h_{n}^{2} e^{-h_{n}^{2} / 2}\right)\right]\right\},
\end{aligned}
$$

where $h_{n}=\sup _{|u| \leq L} g_{n}(u)$. Then the sum over $i$ of the error term in (3.3) can be bounded from above by $c \sqrt{\log (n)} h_{n}^{2} e^{-h_{n}^{2} / 2}$. This error term is negligible provided $h_{n}^{2} \gg 2 \log \log (n)$. To sum up, we have

$$
\begin{aligned}
& \log \prod_{i=i_{0}}^{n} \mathbb{P}\left(X_{i} \leq t_{0}+\frac{g_{n}(u)}{\sqrt{n}}\right) \\
& =\sum_{i \geq i_{0}} \log \int_{-\infty}^{\sqrt{\left|F_{i}^{\prime \prime}\left(t_{i}\right)\right|}\left(g_{n}(u)+\sqrt{n}\left(t_{0}-t_{i}\right)\right)} \frac{1}{\sqrt{2 \pi}} e^{-s^{2} / 2} d s+\mathcal{O}\left((\log (n))^{1 / 2} h_{n}^{2} e^{-h_{n}^{2} / 2}\right) \\
& =\sum_{i \geq i_{0}} \log \int_{-\infty}^{\sqrt{\left|F^{\prime \prime}\left(t_{0}\right)\right|}\left(g_{n}(u)+\sqrt{n}\left(t_{0}-t_{i}\right)\right)+g_{n}(u)\left(\sqrt{\left|F_{i}^{\prime \prime}\left(t_{i}\right)\right|}-\sqrt{\left|F^{\prime \prime}\left(t_{0}\right)\right|}\right)} \frac{1}{\sqrt{2 \pi}} e^{-s^{2} / 2} d s \\
& \quad+\mathcal{O}\left((\log (n))^{1 / 2} h_{n}^{2} e^{-h_{n}^{2} / 2}\right)
\end{aligned}
$$

where we have noted $F^{\prime \prime}\left(t_{0}\right):=-2 / t_{0}^{2}-V^{\prime \prime}\left(t_{0}\right)$. Let us now choose

$$
g_{n}(u):=\frac{f_{n}(u)}{\sqrt{\left|F^{\prime \prime}\left(t_{0}\right)\right|^{3 / 2} t_{0} / 2}} \text { where } f_{n}(u)=\sqrt{2 \log \left(\frac{\left.e^{u} \sqrt{n / 2 \pi}\right)}{\log (n)}\right)} .
$$

Then $f_{n} \rightarrow \infty$ provided $u$ is bounded from below and one can easily check that with such a choice of $f$ all the required estimates on $h_{n}$ hold true. Also there exists a constant $C\left(t_{0}\right)$ such that for any $i \geq i_{0}$

$$
F_{i}^{\prime \prime}\left(t_{i}\right)-F^{\prime \prime}\left(t_{0}\right)=\frac{n-i}{n}\left(C_{0}+o(1)\right) .
$$

The constant $C_{0}$ can be expressed in terms of $t_{0}$ and the derivatives of $V$ : it may be zero. The important fact is that

$$
\left.\sqrt{\left|F^{\prime \prime}\left(t_{0}\right)\right|} \sqrt{n}\left(t_{0}-t_{i}\right)\right)+g_{n}(u)\left(\sqrt{\left|F_{i}^{\prime \prime}\left(t_{i}\right)\right|}-\sqrt{\left|F^{\prime \prime}\left(t_{0}\right)\right|}\right)=\frac{n-i}{\sqrt{n}}\left(\frac{2}{t_{0} \sqrt{\left|F^{\prime \prime}\left(t_{0}\right)\right|}}+o(1)\right) \text {. }
$$

Using now the same arguments as in [18] for the Riemann sum approximation, we again deduce that uniformly on compact subsets,

$$
\log \prod_{i=i_{0}}^{n} \mathbb{P}\left(X_{i} \leq t_{0}+\frac{g_{n}(u)}{\sqrt{n}}\right)=-e^{-u}
$$

Now there remains to consider the smaller integers $i$. Assume now that $i<i_{0}$. The exact critical point $t_{i}$ depends on $n$ and might now be closer and closer to $t_{2}$ where $V^{\prime}\left(t_{2}\right)=0$. We need to control the second derivative of $F_{i}$ to be able to undertake the same Laplace analysis as above.

$$
F_{i}^{\prime \prime}(t)=-V^{\prime \prime}(t)-\frac{2 i-1}{n t^{2}}
$$

By the convexity assumption, we deduce that $t_{i}>t_{2}$ so that the second derivative is negative and bounded from above in the whole interval $\left[t_{2} / 2,2 t_{0}\right]$. From that one can 
deduce that

$$
n\left(F_{i}(t)-F_{i}\left(t_{i}\right)\right)=n \int_{t_{i}}^{t} F_{i}^{\prime}(s) d s=n \int_{t_{i}}^{t} \int_{t_{i}}^{s} F_{i}^{\prime \prime}(u) d u d s \leq-n a\left(t-t_{i}\right)^{2},
$$

by the strict convexity of $V$. In particular for all $i \leq i_{0}$ one has that $t_{i} \leq t\left(i_{0}\right)$ with

$$
\left|t\left(i_{0}\right)-t_{0}\right| \geq C_{5} \sqrt{\frac{\log (n)}{n}}
$$

for some constant $C_{5}>0$. Thus we deduce that

$$
\begin{aligned}
\prod_{i=0}^{i_{0}} \mathbb{P}\left(X_{i} \leq t_{0}+\frac{g_{n}(u)}{\sqrt{n}}\right) & =\prod_{i=0}^{i_{0}}\left(1-\mathbb{P}\left(X_{i} \geq t_{0}+\frac{g_{n}(u)}{\sqrt{(n)}}\right)\right) \\
& =\prod_{i=0}^{i_{0}}\left[1-\frac{\int_{t_{0}+\frac{g_{n}(u)}{\sqrt{n}}}^{e^{n F_{i}(t)} d t}}{\int_{\mathbb{R}^{+}} e^{n F_{i}(t)} d t}\right] \\
& \geq \prod_{i=0}^{i_{0}}\left[1-\left(\sqrt{n}(1 / 2+o(1)) \int_{t_{0}+\frac{g_{n}(u)}{\sqrt{n}}}^{\infty} e^{n F_{i}(t)-n F_{i}\left(t_{i}\right)} d t\right]\right. \\
& \geq \prod_{i=0}^{i_{0}}\left[1-\left(\sqrt{n}(1 / 2+o(1)) e^{-n a\left(t_{0}+\frac{g_{n}(u)}{\sqrt{n}}-t_{i}\right)^{2}}\right]\right. \\
& \geq \prod_{i=0}^{i_{0}}\left[1-\left(\sqrt{n}(1 / 2+o(1)) e^{-a C_{5} c^{2} \log (n)}\right] .\right.
\end{aligned}
$$

Let us now choose $c$ (determining by this way $i_{0}$ ) so that $a C_{5} c^{2}>2$. The latter product goes to 1 as $n$ goes to infinity. This is the needed estimate.

\section{REFERENCES}

[1] Yacin Ameur, Håkan Hedenmalm, and Nikolai Makarov, Fluctuations of eigenvalues of random normal matrices, Duke Math. J. 159 (2011), no. 1, 31-81. MR-2817648[1, 2]

[2] Gérard Ben Arous and Ofer Zeitouni, Large deviations from the circular law, ESAIM Probab. Statist. 2 (1998), 123-134 (electronic). MR-1660943 1

[3] R.N. Bhattacharya and R.R. Rao, Normal approximation and asymptotic expansions, Wiley series in probability and mathematical statistics. Probability and mathematical statistics, Wiley, 1976. 6

[4] Charles Bordenave and Djalil Chafaï, Around the circular law, Probab. Surv. 9 (2012), 1-89. MR-2908617 2

[5] Djalil Chafaï, Nathaël Gozlan, and Pierre-André Zitt, First order global asymptotics for CalogeroSutherland gases, preprint arXiv:1304.7569, 2013. 1.

[6] Ling-Lie Chau and Oleg Zaboronsky, On the structure of correlation functions in the normal matrix model, Comm. Math. Phys. 196 (1998), no. 1, 203-247. MR-1643533 2]

[7] Peter Elbau and Giovanni Felder, Density of eigenvalues of random normal matrices, Comm. Math. Phys. 259 (2005), no. 2, 433-450. MR-2172690 2

[8] Jean Ginibre, Statistical ensembles of complex, quaternion, and real matrices, J. Mathematical Phys. 6 (1965), 440-449. 2

[9] F. Götze and M. Venker, Local Universality of Repulsive Particle Systems and Random Matrices, preprint arXiv:1205.0671, 2012. 4

[10] Alice Guionnet and Ofer Zeitouni, Support convergence in the single ring theorem, Probab. Theory Related Fields 154 (2012), no. 3-4, 661-675. MR-3000558 5

[11] Adrien Hardy, A note on large deviations for 2d Coulomb gas with weakly confining potential, Electron. Commun. Probab. 17 (2012), no. 19, 1-12. 1, 2

[12] J. Ben Hough, Manjunath Krishnapur, Yuval Peres, and Bálint Virág, Zeros of Gaussian analytic functions and determinantal point processes, University Lecture Series, vol. 51, American Mathematical Society, Providence, RI, 2009. MR-2552864]2

[13] Boris Khoruzhenko and Hans-Juergen Sommers, The Oxford Handbook of Random Matrix Theory, ch. 18 Non-Hermitian Random Matrix Ensembles, Oxford University Press, 2011. 2 
[14] Eric Kostlan, On the spectra of Gaussian matrices, Linear Algebra Appl. 162/164 (1992), 385-388, Directions in matrix theory (Auburn, AL, 1990). MR-1148410]2

[15] Manjunath Krishnapur, From random matrices to random analytic functions, Ann. Probab. 37 (2009), no. 1, 314-346. MR-24891672

[16] Valentin V. Petrov, Limit theorems of probability theory, Oxford Studies in Probability, vol. 4, The Clarendon Press Oxford University Press, New York, 1995, Sequences of independent random variables, Oxford Science Publications. MR-1353441 [7]

[17] Dénes Petz and Fumio Hiai, Logarithmic energy as an entropy functional, Advances in differential equations and mathematical physics (Atlanta, GA, 1997), Contemp. Math., vol. 217, Amer. Math. Soc., Providence, RI, 1998, pp. 205-221. MR-1606719 1

[18] Brian Rider, A limit theorem at the edge of a non-Hermitian random matrix ensemble, J. Phys. A 36 (2003), no. 12, 3401-3409, Random matrix theory. MR-1986426 2, 3, 5, 6, 7,12

[19] Brian Rider, Order statistics and Ginibre's ensembles, J. Statist. Phys. 114 (2004), no. 3-4, 1139-1148, MR-20356414 4

[20] Brian Rider and Christopher D. Sinclair, Extremal laws for the real Ginibre ensemble, preprint arXiv:1209.6085, 2012. 2

[21] Edward B. Saff and Vilmos Totik, Logarithmic potentials with external fields, Grundlehren der Mathematischen Wissenschaften [Fundamental Principles of Mathematical Sciences], vol. 316, SpringerVerlag, Berlin, 1997, Appendix B by Thomas Bloom. MR-1485778 2

Djalil Chafaï, Université Paris Dauphine, CEREMAde, IUF, PSL, France

Sandrine PÉché, Université Paris-Diderot, LPMA, France 